\title{
Many-body theory of all-optical quantum well logic gates
}

\author{
M.F. Pereira Jr, M. Prado and R. Sampaio \\ Institute de Fisica, Universidade Federal da Bahia, 40210-340, Salvador B A Brazil \\ Fax: + 557123555 92; e-mail: mauro@fis.ufba.br*
}

\begin{abstract}
A many-body theory, based on the solutions of generalized semiconductor MaxwellBloch equations for coupled-band multiple quantum wells is applied to the description of the optical non linearities responsible for the operation of a resonator as a logical device. The numerical simulation of a NOR gate is in good agreement with experimental realizations.
\end{abstract}

Keywords: optical computing, many-body effects, optical nonlinearities, semiconductor quantum wells.

Paper received 10.03.02; accepted for publication 10.12.02.

\section{Introduction}

Optical computing has been the focus of great interest in the last two decades. It has potential for massive interconnectivity and parallel operations at rates unaccessible to electronics without crosstalk. The Internet and multimedia applications are further fueling an increasing interest on efficient all-optical switching and computing, specially if the information is already being transmitted by light $[1,2]$. Several criteria must be fulfilled in actual devices, e.g., mechanical and operational stability, infinite cascadability, fan-in and fan-out of at least two, input/output isolation, low-switching energy, fast response, good contrast and room-temperature operation. A number of devices with different advantages and drawbacks have been proposed for optical logic gating, where different physical mechanisms in different materials play the dominant role in the device operation, e.g., the interaction of orthogonally polarized beams in a nonlinear Kerr medium [3], coupled gap soliton formation in a fiber Bragg grating pulse compression [4], or optical amplifier-assisted fiber Sagnac Gates [5]. Among the different possibilities, the fast optical non linearities in semiconductor etalons [6-9], are responsible for all-optical logical operations up to $100 \mathrm{Gbits} / \mathrm{s}$ [6], and semiconductorbased devices have the further advantage of lower switching energy. There are several possible choices of semiconductor media. However, high-quality multiple quantum wells (MQW's) can be easily manipulated, costumized and display large room-temperature optical non linearities. So, realistic simulations of MQW based logic devices are necessary, and in order to achieve actual pre- dictability a combination of band structure and manybody effects must be used to describe the MQW optical non linearities [10].

In this paper, to the best of the author's knowledge for the first time, it is demonstrated how a many-body formalism can be applied to the simulation of all-optical NOR gates operation constructed with a MQW structure. The configuration chosen allows for a direct comparison with results in the literature [11], and its simplicity makes it easier to illustrate the power of the numerical algorithm, which can be actually extended and applied to many different and more involved schemes. Furthermore, NOR-gate etalons can be combined to create a complete logical set [12].

\section{Non-linear semiconductor resonator}

Under quasi-equilibrium conditions, corresponding to the device simulated here [11] the nonlinear optical absorption, $\alpha$ and corresponding refractive index change, $\Delta \eta$ at a given energy a; in a MQW characterized by an inversion of population factor, $f_{e v}(k)=f_{e}(k)+f_{v}(k)-1$ is obtained by numerically solving the integral equation for the optical susceptibility, $\chi$ obtained from Semiconductor Bloch Equations, i.e., $\alpha(\omega, N)=(4 \pi \omega) /\left(\Omega n_{b} c\right) \sum_{\vec{k} e v} \mu_{k e v}^{*} x_{e v}^{\prime \prime}(k, \omega)$

$$
\begin{aligned}
& \left(\omega-\Delta_{c v}(k)\right) \chi_{e v}(k, \hbar \omega)= \\
& =f_{e v}(k)\left[\mu_{e v}(k)+\sum_{\vec{q} \neq 0} W_{\vec{k}-\vec{q}}\right] \chi_{e v}(q, \hbar \omega) .
\end{aligned}
$$

\footnotetext{
*Presenet address: NMRC, Lee Maltings, Cork, Ireland, e-mail: mpereira@nmrc.ie; fax: +353214904058
} 


\section{M.F. Pereira et al.: Many-body theory of all-optical quantum well logic gates}

Here $W$ is the screened potential, $\Delta_{c v}(k)=E_{e}(k)-E_{v}(k)$, $E_{\lambda}$ and $f_{\lambda}$ are the renormalized energy and occupation factor which characterize the sub band $\lambda, n_{b}$, is the background refractive index, $\Omega$ is the sample volume, $\mathrm{c}$ is the velocity of light in vacuum, and the indices $e, v, e=\mathrm{e}_{1}, \mathrm{e}_{2}$, $\ldots, v=v_{1}, v_{2}, \ldots$, label the multiple electron and hole sub bands and incorporate the total angular momentum $z$ projection for electrons and a block diagonalization index which characterizes the coupled heavy- and lighthole bands. For more details see [10]. The refractive index change $\Delta n$ with respect to the background index can be either directly computed from the real part of $\chi$ or by means of a Kramers-Kronig transformation with the absorption changes as input.

Note that more advanced numerical schemes are possible for either Semiconductor-Bloch or Bethe-Salpeter Equations [13,14], but the formalism chosen has all the required elements for the consistent solution of the problem, $\chi$ " denotes the imaginary part of $\chi$. The computed microscopic optical response of the medium is then incorporated in the (macroscopic) Maxwell's equations, which are solved in the paraxial approximation [15]. The etalon transmission at steady state then reads [16]

$$
I_{t}=\frac{I_{0} T^{2}}{\left(e^{a(\omega, N) L / 2}-\mathrm{Re}^{-a(\omega, N) L / 2}\right)^{2}+4 R \sin ^{2}\left(\omega L / c\left[n_{b}+\Delta n(\omega, N)\right]\right)}
$$

Here $R$ and $T=(1-R)$ denote respectively the reflectivity and transmitivity of the etalon. For thin samples like those considered here, diffusion effects can be neglected. Thus, introducing the average intensity inside the resonator, $I=I_{t}(1+R) / T$, and the carrier recombination lifetime $r$, the average carrier density inside the resonator, generated by a pump light field, can be approximated by the rate equation $d N / d t=-N / \tau+\alpha(\omega, N) I / \omega$. Figure 1 shows the local absorption $(a)$ and refractive index changes (b) predicted by the theory for a $76 \AA \mathrm{GaAs}$ $\mathrm{Al}_{0.37} \mathrm{Ga}_{0.63}$ quantum well at $300 \mathrm{~K}$. and as in the experimental paper, the electron-heavy hole exciton appears around $840 \mathrm{~nm}$ and the absorption there is of order $1 \mu \mathrm{m}^{2}$. No effort has been made to fit the experimental data by adjusting material material parameters and band gaps. The values used are the same as in [10] where a different set of GaAs-AlGaAs and $\mathrm{InGaAs}-\mathrm{AlGaAs}$ has been rather successfully described. The design parameters, like well width, alloy concentration, number of wells and operating wavelength for the etalon are the nominal values of [11]. The local absorption as a function of carrier density (a) and corresponding refractive index change (b) at $873.1 \mathrm{~nm}$ are depicted in Fig. 2. The local induced detuning in the resonator formula (dot-dashed) and the normalized transmission, $I_{t} /\left(I_{0} T^{2}\right)$, of a two-wavelength NOR etalon gate (solid) made with 63 periods of $76 \AA$ GaAs and $81 \AA \mathrm{Al}_{0.37} \mathrm{Ga}_{0.63}$ As barriers are depicted in Fig. 3. Experimental data extracted from [11] is given as symbols in the inset. The first dip has been normalized to fit the generated data. The real-time NOR gate operation simulated here can be summarized as follows.

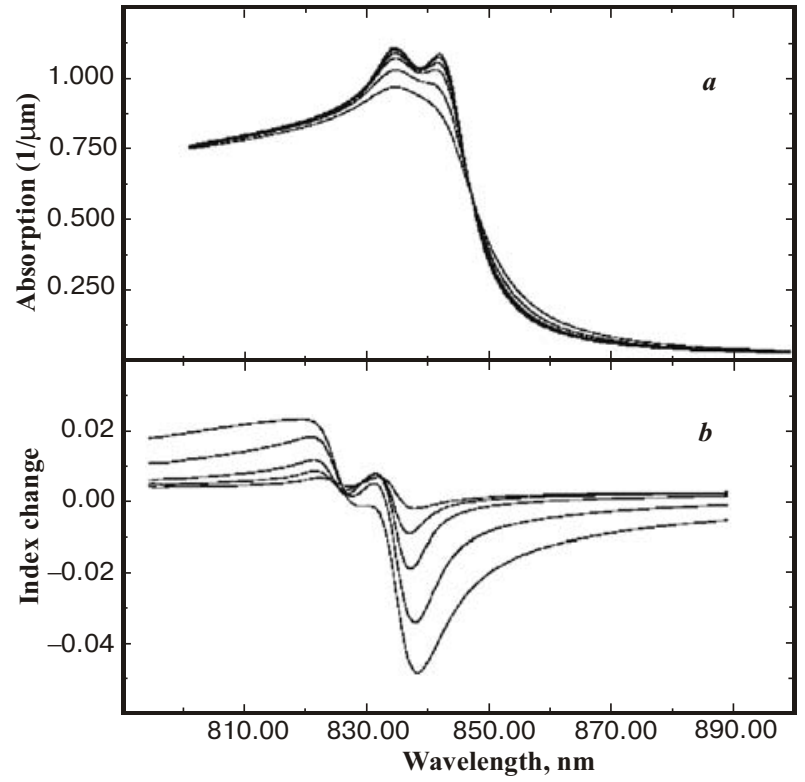

Fig. 1. $a$ - Absorption predicted by the theory for a $76 \AA \mathrm{GaAs}-$ $\mathrm{Al}_{0.37} \mathrm{Ga}_{0.63}$ quantum well at $300 \mathrm{~K}$. The carrier densities are given, from top to bottom, by $N=0.0,0.5,1.0,2.0,5.0,10.0 \times 10^{17} \mathrm{~cm}^{-3}$; $b$ - Corresponding refractive index changes with respect to the background due to the charged carriers, i.e., from top to bottom, $N=0.5,1.0,2.0,5.0,10.0 \times 10^{17} \mathrm{~cm}^{-3}$.

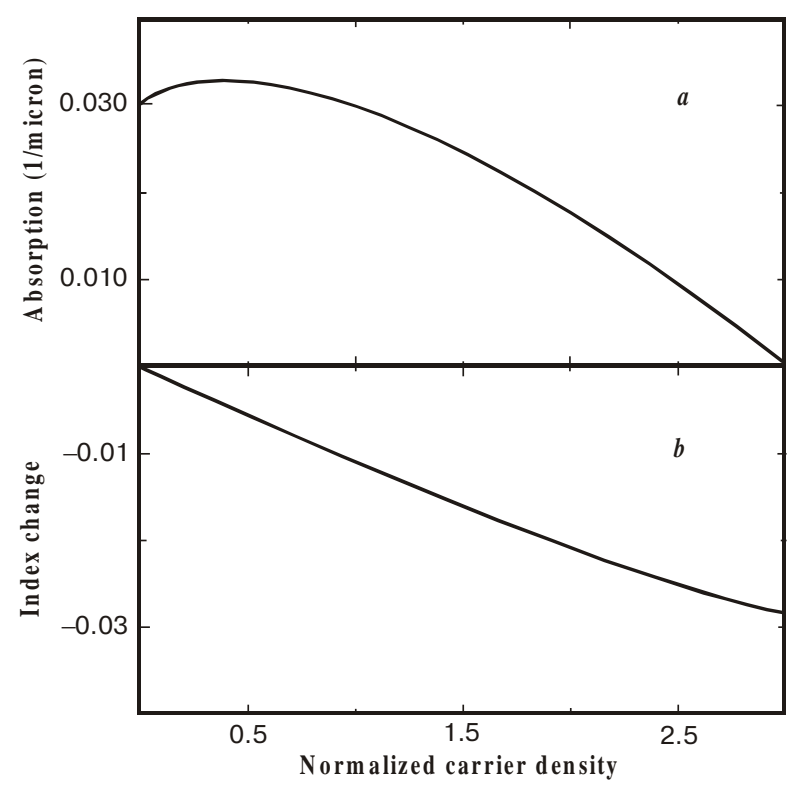

Fig. 2. $a$ - Local TE absorption of the target wavelength $873.1 \mathrm{~nm}$ in a $7.6 \mathrm{~nm} \mathrm{GaAs}-\mathrm{Al}_{0.37} \mathrm{Ga}_{0.63} \mathrm{As}$ quantum well at $300 \mathrm{~K}$ in as a function of the pump generated carrier density $1 \times 10^{18} \mathrm{~cm}^{-3} ; b-$ Corresponding absolute value of the refractive index change with respect to the background index due to the presence of carriers. 


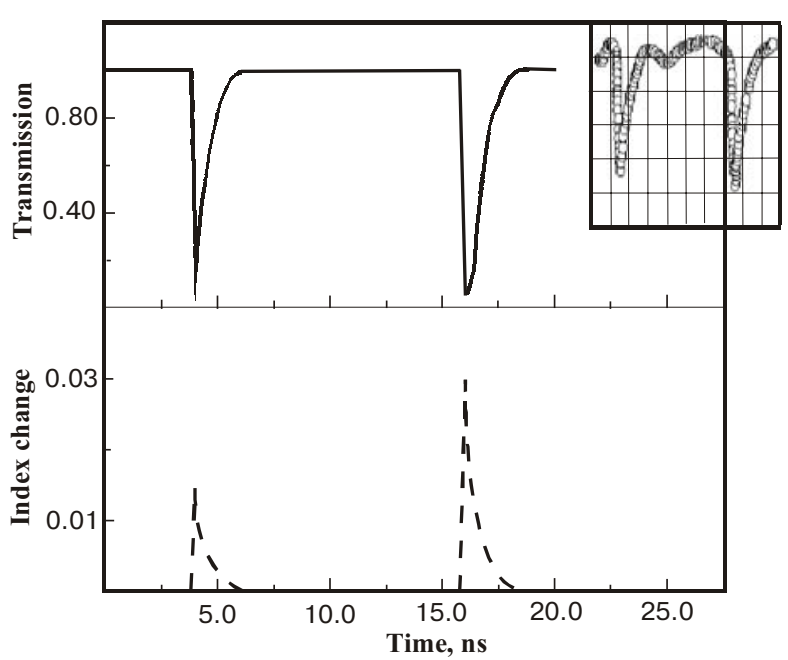

Fig. 3. $a$ - Non linear transmission, $I_{t} /\left(I_{0} T^{2}\right)$, spectra (from Eq. 2) at $300 \mathrm{~K}$ of a clocked probe beam (solid) which arises as a consequence of the $(b)$ refractive index change at $873.1 \mathrm{~nm}$ (dot-dashed) generated by pump beams in a stack of 63 wells separated by $8.1 \mathrm{~nm}$ barriers. The symbols in the inset are experimental data extracted from [11]. (oscilloscope image). The $\mathrm{x}$-y grid corresponds to $2 \mathrm{~ns}$ and $5 \mathrm{mV}$.

A clocked probe beam at resonance with the cavity in the low absorption range of the optical spectrum hits the gate. If no other beams are present, the transmitted intensity is high. However, the presence of either one or two pump pulses in the high absorption region of the quantum spectrum generates a high density of carriers. Those carriers in turn will change the refractive index at the probe frequency and the clocked signal will be transmitted with low intensity. Note that the photo-generated carriers relax into a quasi-equilibrium state within a few hundred femtoseconds after excitation by the short pump pulses. Under these conditions, the probe finds an etalon nonlinearity which depends exclusively on the density of generated carriers $N$. The many-body effects responsible for device operation are then given by bandgap shrinkage, screening of the Coulomb interaction and Pauliblocking. As a matter of fact, the carrier density is not uniform in the excited media. However, the good agreement between theory and experiment obtained shows that for the conditions considered, a uniform density can be used as a first approximation. For the carrier densities chosen in the calculations, a contrast with one input of $\approx 6.2: 1$ is found tobe in very good agreement with the experimental value appears to be approximately over $6: 1$. Limitations in the detector used did not allow the observation of maximum contrast. Note that, with two pulses, the contrast is a little better and the recovery takes a longer time, as in the experiments, further demonstrating the predictability power of the approach, which is being currently extended to include dynamical screening and non-diagonal dephasing effects, that will make it more powerful, by generalizing a recent approach for bulk systems [17].
In summary, a many-particle theory for optical absorption and refractive index change in coupled band semiconductor quantum wells combined with the paraxial Maxwell equations has been applied to the study of nonlinear semiconductor resonators. The resulting equations are capable of realistically simulating the operation of semiconductor all-optical gates. The algorithm can be used in the design and simulation of optoelectronic devices, exploiting fundamental mechanisms, which can only be described by a combination of Coulomb, band structure engineering and cavity resonator effects. The controllable non linear phenomena can be adapted to many different semiconductor-based configurations.

Research supported by the Conselho Nacional de Pesquisas (CNPq) of Brazil. M. Prado and R. Sampaio acknowledge PIBIC Fellowships.

\section{References}

1. C. Coriasso, D. Campi, C.Cacciatore, L. Faustini, A. Stano, and C. Rigo, "All-optical switching and pulse routing in a distributed-feedback waveguide device", Opt. Lett., 23, 183 (1998).

2. Sahlen, U. Olin, G. Landgren and M. Rask, "Subnanosecond absorptive switching in GaAs etalons", Journal de Physique, Colloque C2, 49, C2-19 (1988).

3. M. Zitelli, E. Fazio, and M. Bertolotti, "All-optical NOR gate based on the interaction between cosine-shaped input beams of orthogonal polarization, J. Opt. Soc. Am. 16, 214 (1999).

4. D. Taverner, N. G. R.Broderick, D. J. Richardson, M. Ibsen and R. I. Laming, All-Optical "AND" Gate Based on Coupled Gap Soliton Formation in a Fibre Bragg Grating Pulse Compresion in fiber Bragg gratings, Opt. Lett. 23, 259 (1998).

5. T. Houbavlis, K. Zoiros, A. Hatziefremidis, L. Avramopoulos, L. Occhi, G. Guekos, S. Hansmann, H. Burkhard, and R. Dall'Ara, "lOGbit/s all-optical Boolean XOR with SOA fibre Sagnac gate", Electron. Lett. 35, 1650 (1999).

6. K.L. Hall, K.A. Rauschenbach, "100 Gb/s bitwise logic", Opt. Lett. 231271 (1998).

7. A.D. Ellis, D.M. Pattrick, D. Flannery, R.J. Manning, D.A.O. Davies, and D.M. Spirit, "Ultra-high-speed OTDM networks using semiconductor amplifier-based processing nodes", J. Lightwave Technol. 13, 2099 (1995).

8. M. Eiselt, W. Pieper, and H.G. Weber, "SLALOM: Semiconductor laser amplifier in a loop mirror", J. Lightwave Technol. 13, 2099 (1995).

9. J.P. Sokoloff, P.R. Prucnal, I. Glesk, and M. Kane, "A terahertz optical asymmetric demultiplexer (TOAD)", IEEE Photonics Technol. Lett. 5, 787 (1993).

10. Theory of nonlinear absorption in coupled band quantum wells with many-body effects,

M.F. Pereira Jr., R. Binder and S.W. Koch, Appl. Phys. Let. 64, 279 (1994).

11. J.L. Jewell, Y.H. Lee, M. Warren, H.M. Gibbs, N. Peyghambarian, A.C. Gossard and W. Wiegmann, 3-pj, 82$\mathrm{MHz}$ optical logic gates in a room temperature GaAs-AlGaAs multiple-quantum-well etalon, Appl. Phys. Lett. 46, 918 (1985).

12. A. Huang, "Parallel algorithms for optical digital computers", in 10th International Optical Computing Conference, IEEE Computer Society Press, Silver Spring Maryland, 1983.

13. M.F. Pereira Jr. and K. Henneberger, Microscopic Theory for the Influence of Coulomb Correlations in the Light-Emission Properties od Semiconductor Quantum Wells, Phys. Rev. 58, 2064 (1998). 
M.F. Pereira et al.: Many-body theory of all-optical quantum well logic gates

14. A. Girndt, F. Jahnke, A. Knorr, S.W. Koch, and W.W. Chow, Multi-Band Bloch Equations and Gain Spectra of Highly Excited II-VI Semiconductor Quantum Wells, Phys. Stat. Solidi ? 202, 725 (1997).

15. M. Lax, W.H. Louisell and W.B. Mcknight, From Maxwell to paraxial wave optics, Phys. Rev. A 11, 1365 (1975).
16. S.W. Koch, "Optical instabilities in semiconductors: Theory", in "Optical Nonlinearities and Instabilities in Semiconductors", Edited by H.M. Haug, Academic, New York, 1988.

17. G. Manzke, Q.Y. Peng, K. Henneberger, U. Neukirch, K. Hauke, K. Wundke, J. Gutowski, and D. Hommel, Density Dependence of the Exciton Energy in Semiconductors, Phys. ev. Lett. 22, 4943 (1998). 